\title{
Surfaces
}

\section{LES MURMURES DE LA MACHINE : LIRE À TRAVERS LE BRUIT DE FOND DE DON DELILLO}

\section{Bertrand Gervais}

Volume 4, 1994

SUR L'ÉPISTÉMOLOGIE DE LA GUERRE FROIDE

ON COLD WAR EPISTEMOLOGY

URI : https://id.erudit.org/iderudit/1064961ar

DOI : https://doi.org/10.7202/1064961ar

Aller au sommaire du numéro

Éditeur(s)

Les Presses de l’Université de Montréal

ISSN

1188-2492 (imprimé)

1200-5320 (numérique)

Découvrir la revue

Citer cet article

Gervais, B. (1994). LES MURMURES DE LA MACHINE : LIRE À TRAVERS LE BRUIT DE FOND DE DON DELILLO. Surfaces, 4.

https://doi.org/10.7202/1064961ar
Résumé de l'article

Pour expliquer la convergence de la théorie et de la pratique qui caractérise la réception critique du roman White Noise de Don DeLillo, l'auteur fait appel à la notion de communauté interprétative de Stanley Fish. Il examine ensuite certains des dispositifs par lequel le roman s'inscrit, en toute nécessité, dans le courant esthétique postmoderne. 


\title{
LES MURMURES DE LA MACHINE: LIRE À TRAVERS LE BRUIT DE FOND DE DON DELILLO
}

\section{Bertrand Gervais}

\section{RÉSUMÉ}

Pour expliquer la convergence de la théorie et de la pratique qui caractérise la réception critique du roman White Noise de Don DeLillo, l'auteur fait appel à la notion de communauté interprétative de Stanley Fish. Il examine ensuite certains des dispositifs par lequel le roman s'inscrit, en toute nécessité, dans le courant esthétique postmoderne.

\begin{abstract}
To explain the convergence of theory and practice which caracterizes the critical reception of Don DeLillo's novel, White Noise, the author introduces Stanley Fish's notion of interpretative community. He then examines some of the unmistakable features by which the novel signals its ties to the current postmodern aesthetics.
\end{abstract}

Were people this dumb before watching television?

(White Noise ) 
Modernism insisted that cognition can grow independently,

television

has

demonstrated

that it can

also

decrease

collectively.

(Charles

Newman, The Postmodern

Aura)

Certaines lectures semblent aller de soi. Le texte colle tellement bien à l'esprit du temps, il répond tellement bien aux attentes du lecteur, que les résultats de leur rencontre sont d'une grande homogénéité et, par suite, d'une semblable prévisibilité. Malgré son hétérogénéité fondamentale, ses allures de grand centre d'achat de la culture américaine contemporaine, le roman White Noise de Don DeLillo[1](traduit sous le titre de Bruit de fond) fait l'objet de telles lectures. Non pas qu'il soit un exemple de littérature populaire, appelant une lecture sérielle, axée sur la répétition, le formulaïque[2] au contraire, le roman ferait plutôt partie de l'avant-garde littéraire américaine, mais ses interprétations les plus fréquentes, à l'heure actuelle, n'ont de cesse de réclamer et d'illustrer la dimension postmoderne de ses principaux dispositifs. Le roman est l'objet d'un consensus, c'est une œuvre postmoderne, et les interprétations qui en sont faites respectent les principales composantes de cette esthétique, au point souvent de se redoubler. Ce qu'on dit de White Noise se ressemble et s'assemble, comme s'il n'était pas possible de dire ou de faire autrement, comme si l'offre répondait tout à fait à la demande. Cette convergence interprétative ne doit cependant pas surprendre: le roman semble être une parfaite illustration, ironie incluse, des théories postmodernes pratiquées et discutées aux ÉtatsUnis, une fictionnalisation articulée sur ses préceptes, valeurs et savoirs. Cette convergence n'est donc pas un fait du hasard, mais constitue une régularité dont j'aimerais démontrer quelques-uns des principaux mécanismes, parmi lesquels le traitement de l'histoire ou, pour être plus précis, de la guerre froide, de même que de la technologie, de la vidéosphère et de son régime sémiotique bien précis. 


\section{La grange sémiotique}

Don DeLillo fait partie de ces auteurs qui, avec John Barth, Thomas Pynchon, William Gaddis, Robert Coover, Donald Barthelme et de nombreux autres, sont identifiés comme étant résolument postmodernes. L'ensemble de son œuvre cadre bien avec cette perspective d'interprétation et White Noise, qui date de 1984, n'est pas différent des autres romans à cet égard. Les principaux essais qui lui ont été consacrés concordent sur ce point. De par l'importance accordée au simulacre, qui règne en roi, à la société de consommation et de communication, ici parodiée, à la crise du sujet, dont les insécurités fondamentales ne peuvent plus être résolues sinon de façon artificielle, à la paralysie des politiques sociales et économiques, l'écrasement de l'Histoire, l'hégémonie de la technologie, l'existence en périphérie, la prépondérance de la culture populaire, les transformations de la famille, etc., il s'agit bien d'un texte postmoderne.

Ainsi, dans la série des "New Essays", les auteurs du collectif sur White Noise, édité par Franck Lentricchia[3], sont unanimes à lui reconnaître ce statut. Les opinions convergent, que ce soient celles de l'éditeur lui-même, dans son introduction et sa propre contribution, que celles de Ferraro, Cantor, ou Valdez Moses. On retrouve la même affirmation dans des articles de John Frow, de Leornard Wilcox, de Noel King, de Paula Bryant[4], de Michael Messmer[5], etc. On associe régulièrement DeLillo à Barthes, Baudrillard, Heidegger et Jameson; on négocie les divers virages postmodernes du texte avec doigté et on discute continuellement du statut de l'œuvre: jusqu'à quel point, comment, pourquoi, de quelle façon, selon qui, quand, où et à quel niveau est-elle postmoderne? Noel King, par exemple, s'interroge:

What exactly is the relation of White Noise to the category of the postmodern? Is it to be called a postmodern novel because it talks about postmodern sunsets, semiotics and simulacra? Is it postmodern in the sense that the novels of Pynchon, Gaddis and Coover are termed postmodern? Or is it, rather, a slyly modernist meditation on postmodern themes?[6]

Donnant un autre son de cloche, Tom LeClair, dans un essai qui décrit les relations qu'entretient la production romanesque de DeLillo avec la théorie des systèmes de von Bertalanffy, avance que White Noise n'est pas postmoderne, mais plutôt "re-modern", un nouvel avatar du modernisme.[7] Est-ce donc un roman postmoderne, moderne ou re-moderne portant sur des thèmes postmodernes? Il est difficile sinon superflu d'y répondre. Ce qui est 
facile à remarquer, par contre, c'est l'homogénéité du regard, de même que les procédés, stratégies ou dispositifs du texte retenus.

Une des scènes citées avec le plus de régularité, et qui survient au tout début du roman, concerne une grange dont la réputation est une pure fabrication, puisqu'elle serait la grange "la plus souvent photographiée en Amérique". Il s'agit d'une de ces attractions dont les États-Unis ont la recette et qui consiste en un site touristique sans valeur réelle et dont la popularité repose uniquement sur un campagne publicitaire savamment menée, en l'occurrence une série d'affiches routières annonçant le site en question. C'est ainsi que dans le Dakota du sud, une simple pharmacie, du nom de Wall Drug, dans un petit village à l'entrée du Badlands National Parc, un coin perdu s'il en fût jamais un, a réussi à devenir une attraction touristique importante, attirant à elle seule des centaines de milliers de voyageurs. Annoncée par des affiches sur plus de deux cents kilomètres, qui finissent par attirer l'attention par leur démesure et leur persistance, la pharmacie s'est transformée d'une simple halte routière en une attraction, avec restaurant, pouvant asseoir jusqu'à 410 touristes, galerie d'art western, magasins de toutes sortes, etc. On n'a aucune raison d'être là, sauf d'avoir été happé par l'insistance des affiches. Et au sortir de la pharmacie, on reçoit gratuitement un collant pour son pare-choc, qui annonce triomphalement "I've been to Wall Drug".

Dans White Noise, le site annoncé par les affiches est donc "THE MOST PHOTOGRAPHED BARN IN AMERICA" (12; les majuscules sont dans le texte). Annoncée par une série de cinq pancartes, elle n'existe en tant que site que par l'intérêt générée par la publicité. Quand le narrateur et héros du roman, Jack Gladney, et son copain Murray s'y rendent, ils comprennent rapidement, à voir l'autocar de touristes et les quarante automobiles garées, que c'est leur présence à eux tous qui crée l'événement, qui constitue le site. Murray explique: "We're not here to capture an image, we're here to maintain one. Every photograph reinforces the aura" (12). Or, si l'exemple de cette grange est cité à répétition -- et il le sera par des critiques comme Franck Lentriccia, Leonard Wilcox, Noel King, John Frow, pour ne nommer que ces quatre-là -- si donc cet exemple est mécaniquement sinon machinalement reproduit (et je participe bien malgré moi à ce processus), c'est qu'il apparaît comme un jeu sur les propositions, tout à coup inversées, de Walter Benjamin sur la reproduction des objets d'arts, de même qu'une reprise des développements plus récents de Baudrillard sur les simulacres.

La grange, déjà symbole dans le texte, puisque son apparition dans le roman est préalablement médiatisée par sa mise en site et son interprétation, le devient doublement par ces lectures qui la rendent encore plus emblématique d'un discours, d'un savoir. Elle est le signe, et à ce titre elle acquiert un nouvel aura, du postmodernisme: une des traces laissées par ce roman qui agit comme un véritable petit Poucet. 
Comme semble le craindre Robert Dion, "L'histoire de la postmodernité pourrait être vue comme celle de l'avènement simultané -- et autoproclamé -- d'une littérature, d'une critique et d'une théorie postmodernes" ("Une critique" 89). C'est le cas avec la grange de White Noise: à la fois élément d'une fiction, argument d'une critique et application d'une théorie. Dion met en doute ce qui apparaît une vision réductrice et caricaturale de la critique postmoderne, qui ne serait plus "en somme, que le relais publicitaire d'une littérature du n'importe quoi"[8], le règne du "anything goes" dont la conséquence ultime serait la fin de la critique, fondée sur sa renonciation à être justement critique.

Mais il y a une autre façon de comprendre cette correspondance entre littérature, critique et théorie, ce zeitgeist qui rabat diverses pratiques discursives les unes sur les autres. Elle ne signale pas tant la fin de la critique ou sa subordination, que l'existence et le fonctionnement de ce que Stanley Fish nomme une communauté interprétative. Le postmodernisme en littérature est une communauté interprétative, c'est-à-dire, au sens fort, l'ensemble de ceux et celles qui partagent les mêmes stratégies interprétatives non pas tant pour lire que pour écrire les textes, pour établir leurs propriétés et leur attribuer des intentions.[9] Les communautés interprétatives opèrent en imposant leur conception de la littérature, la façon dont les textes doivent être compris et lus, de même que les stratégies permettant d'établir leur signification. Elles assurent la stabilité de certaines interprétations et, par suite, favorisent l'utilisation de procédés dont les effets seront ultérieurement reconnus.

White Noise et ses critiques participent de la même communauté: ils partagent les mêmes conceptions de la littérature et de ses poétiques les plus récentes, du monde contemporain et de ses complexités, du rapport du sujet à la société, à l'histoire, à la technologie. C'est ce qui explique la convergence interprétative marquée qui les caractérise, de même que la disponibilité du texte lui-même, lequel rend possible et favorise cette convergence. Elle ne rend pas le roman moins intéressant, elle lui confère plutôt une grande efficacité, une pertinence immédiate, qu'il perdra peutêtre lorsqu'il sera saisi par une autre communauté interprétative. Entre temps, j'aimerais examiner certains des dispositifs du texte qui inscrivent cette disponibilité.

\section{Le faux-semblant}

White Noise se laisse lire comme un roman postmoderne car il reprend sur le mode fictionnel les principales conceptions et normes de cette esthétique qui se voient pour ainsi dire confirmées dans leur agir. Le roman ne 
comporte aucune intrigue globale. Il y a bien trois parties, se distribuant en 40 chapitres, mais les liens qui les unissent ne dépendent d'aucune scénarisation soutenue. Les pages du roman sont remplies de ces petits événements de la vie quotidienne: l'épicerie, le travail, les maladies des enfants, les amis, la peur de la mort et les désastres écologiques. Il est constitué d'éléments hétérogènes, dispersés, de fragments de vie et de réflexions philosophiques, du bourdonnement d'une société de consommation littéralement noyée dans un trop plein d'informations. L'action se passe à la périphérie, dans la petite ville universitaire de Blacksmith, et met en scène une famille qui ne déplairait ni à Dan Quayle ni à Murphy Brown. Elle est peut-être complète, avec un père et une mère -Jack Gladney, le narrateur, et sa femme Babette -- mais les enfants proviennent de mariages antérieurs et leur nombre reste longtemps indéterminé. C'est une famille à géométrie variable.

Si c'est un roman sans histoire, c'est un roman aussi où l'Histoire fait l'objet d'un aplatissement et d'une banalisation presque totale. Comme au magasin d'alimentation du roman, sorte de foire débordante, grande surface aux allées interminables et complexes comme un labyrinthe borgésien où on retrouve de tout, des denrées du monde entier perdant toute valeur du fait de l'arbitraire de leur contiguïté[10], l'univers de Gladney mêle les temps, les périodes, se les approprie dans le mouvement d'une mixtion improbable, rendue de ce fait insipide. L'Histoire est un produit qui s'échange, se vend, s'exploite et grâce auquel on peut faire une carrière, surtout universitaire. Ainsi en est-il des études hitlériennes, dont Jack Gladney est le grand spécialiste, étant l'inventeur et le directeur du département qui les dirige. Pour lui, le choix d'Hitler n'est pas une question éthique ou idéologique, mais découle simplement d'un opportunisme académique. Dans le vaste horizon des études culturelles et des champs d'étude contemporains, il fallait trouver la perle rare, l'objet neuf, la terra incognita, et c'est ce qu'a fait Gladney.

I invented Hitler studies in North America in March of 1968. It was a cold bright day with intermittent winds out of the east. When I suggested to the chancellor that we might build a whole department around Hitler's life and work, he was quick to see the possibilities. It was an immediate and electrifying success. (4)

Voilà, c'est tout. Aucune justification politique, historique, sociale (à noter que ça prenait un chancelier pour en apprécier un autre...). Ce sera Hitler, comme ç'aurait pu être Elvis Presley ou les extra-terrestres. Tous les sujets se valent: leur importance provient de leur excentricité plutôt que d'une qualité intrinsèque. D'ailleurs Murray, l'ami de Gladney, tentera sa chance avec Elvis, avant de se rabattre sur un séminaire portant sur les accidents d'auto au cinéma.[11] C'est Hitler donc, parce que ça n'avait pas encore été fait et que le sujet fascine le public américain, un peu comme la pornographie (à laquelle DeLillo avait déjà associé le führer, dans Running 
Dog). Le roman, cependant, n'est pas une adhésion à l'hitlérisme; il en est simplement la commercialisation.[12] Les études hitlériennes forment une coquille vide, qu'on ne pénètre jamais, mais qui est manipulée, un peu comme un livre, entre les mains de libraires. Gladney lui-même traite sa matière comme un spectacle, travestissement inclus. Bien qu'il en soit l'initiateur, il ne parle pas l'allemand qu'il tentera d'apprendre tout au long du roman. Il porte une toge, ce que son rang universitaire lui permet, et n'enseigne qu'avec des lunettes fumées qui ajoutent à sa prestance. Il a pris du poids, de façon à remplir son personnage; il a changé son nom en ajoutant des initiales à son prénom, passant de Jack à J. A. K. Gladney. Le résultat est un simulacre plus vrai que nature ("I am the false character that follows the name around" (17)), tout à l'image de ce savoir qui n'en est pas un.

Hitler est d'ailleurs perçu en termes de fiabilité et de solidité ("--How is Hitler? --Fine, solid, dependable".(89)), comme s'il s'agissait d'un produit quelconque, fait pour durer. Une fois choséifié et réduit à ses traits les plus bénins - -- son charisme, la théâtralisation de son pouvoir --, Hitler devient l'objet d'une appropriation: du réel et de l'historique, il passe dans l'imaginaire où il devient une sorte de pâte à modeler s'adaptant à tous les désirs.[13] Il est l'ennemi par excellence, puisqu'il est l'ennemi qui a été vaincu: il permet donc à qui s'en empare d'assurer sa domination et de se poser en héros justicier. Il est important en ce qu'il assure une vision du monde, un ordre mondial.

On pourrait dire que cette assimilation participe d'un processus plus large: l'intégration de la guerre froide à même le tissu de l'univers de Blacksmith. White Noise n'est pas un roman de la guerre froide, mais il participe à sa relance, celle qui est apparue avec l'arrivée au pouvoir des Républicains et de Ronald Reagan et qui est maintenant déclarée terminée, depuis la chute du mur de Berlin et l'éclatement de l'Union soviétique. Cette permanence de la guerre froide pourrait être comparée, en fait, au comportement d'un produit radioactif, car si sa durée initiale fut plutôt brève, sa demi-vie est beaucoup plus longue. Elle s'éternise et, ce faisant, elle s'est immiscée dans de nombreux aspects de la vie. Ainsi, la guerre froide n'est pas thématisée dans White Noise, mais sa tension s'y fait sentir de façon implicite. Elle est présente dans les études hitlériennes de Gladney, liées par définition à la seconde guerre mondiale, à la militarisation et aux conflits mondiaux. Elle est présente dans les exercices d'évacuation qui sont effectués à l'école et en ville et auxquels participent les enfants de Gladney. Il n'y a plus de peur atomique, mais ce danger a simplement été changé pour un autre, de nature toxique. L'ennemi n'est pas là, mais l'habitude de sa menace persiste, signe qu'il a été assimilé. Elle est présente aussi à travers tous ces personnages secondaires, les femmes ou les maris des femmes de Gladney, qui font de l'espionnage: l'une travaille pour la CIA, l'autre a un mari qui déstabilise des régimes communistes. Quand il n'y a plus de menace, il faut en créer une car c'est dans l'adversité qu'on retrouve sa raison d'être. Un ami de Heinrich, Mercator, s'entraîne ainsi pour briser un record d'endurance et 
rester le plus longtemps possible dans une pièce fermée pleine de serpents venimeux.

Sous le calme apparemment plat de la petite vie d'une ville universitaire, couve donc une tension qui a pour origine et justification le maintien d'une guerre froide. Une guerre intériorisée, absorbée comme un médicament, qu'on ne voit plus par conséquent mais dont les effets se font toujours sentir. Un froid imaginaire, comme une peur de la mort qui ne disparaît plus, sauf à prendre quelques Dylar (l'ancêtre du Prozac?), la drogue miracle du roman, qu'une compagnie pharmaceutique développe en secret, à l'abri justement de tout espionnage. La guerre froide est l'horizon nécessaire du roman.

\section{Sphères à l'œuvre}

Le dernier trait sur lequel j'aimerais maintenant m'arrêter concerne le rapport complexe que le roman entretient avec les technologies, surtout celles de la communication. Comme le veut la doxa postmoderne, l'univers du roman est un monde tout en surface, où la technologie est condition même de l'expérience humaine, son mode privilégié de connaissance, de l'autre tout comme de soi. C'est un monde régi par des écrans cathodiques, les télécommunications, des médias de toutes sortes. Radios, télévisions, émissions diverses et tabloïds produisent ce bruit de fond, ce white noise, dans lequel tous baignent et à travers lequel ils tentent, souvent sans succès, de se comprendre. Cette société technologique est, aux dires de plusieurs, un régime "technico-sémiotique", où les images et les représentations remplacent l'expérience directe. Ce régime, c'est la vidéosphère, selon l'expression utilisée récemment par Régis Debray. Dans son Cours de médiologie générale, puis surtout dans Vie et mort de l'image et tout récemment dans L'état séducteur[14], Debray propose une étude non pas de la signification, des énoncés et de leurs contenus, mais des messages eux-mêmes, des modes de transmission ou des médiations, et de leur impact sur une société. C'est ce qu'il nomme la médiologie. Je ne veux pas m'attarder longuement à cette discipline en devenir, à ses multiples emprunts, à sa méthodologie un peu molle, si ce n'est pour en retenir l'esprit: une attention à la matérialité même des systèmes de communication et la notion centrale de médiasphère qui en est le corollaire. L'idée même de médiasphère découle du principe qu'on ne peut "séparer une opération de pensée, à quelque époque que ce soit, des conditions techniques d'inscription, de transmission et de stockage qui la rendent possible" (Médiologie générale 229). Ce n'est pas un simple cas de logique à la McLuhan, où le médium serait tout bonnement le message; mais la reconnaissance d'une interaction complexe où la nature même du médium affecte la pensée, jusqu'à infléchir sa course. Les modes de transmission, selon cette perspective, n'accompagnent pas la pensée, mais la modèlent bel et bien, lui donnant forme et horizon. De fait, selon Debray, "le système 
dominant de conservation des traces (saisie, stockage et circulation) sert de noyau organisateur à la médiasphère d'une époque donnée dans une société donnée" (Médiologie générale 229). Trois médiasphères sont identifiées dont la succession correspond au développement historique des moyens de transmission. La logosphère est la première sphère et elle coïncide avec l'invention de l'écriture. Elle est suivie par la graphosphère qui se construit, elle, sur l'imprimerie, le livre. Le dernier âge, celui de l'électron, de l'audiovisuel, est la vidéosphère. Chaque âge a sa particularité, sa dominante, ses polarités.

Le dernier, présent dans White Noise, se caractérise par le visuel.[15] Pas l'icône, pas la toile ou encore l'image de cinéma, qui existent toutes physiquement -- sur ruban, canevas ou matériau primaire --, mais l'image vidéo, immatérielle, un signal électrique balayant un moniteur, dont nous avons à reconstituer nous-même les résultats, un modèle logicomathématique provisoirement stabilisé. Ce n'est plus un univers de la projection, de la lumière réfléchie du dehors, mais de la diffusion, de la lumière émise par l'écran.[16] La vidéosphère implique un rythme accéléré, celui de l'instantanéité et, du même coup, une hégémonie, celle de son économie symbolique. L'espace-temps qu'elle suscite s'organise autour d'émissions de toutes sortes, d'un surplus de communications qui se complètent, se chevauchent et se contredisent tout en même temps, en s'imposant comme ambiance. Ce n'est pas pour rien que le roman s'intitule White Noise: plus qu'un simple bruit de fond, il est l'opposé d'un son pur, d'une tonalité unique, à savoir un son qui a toutes les tonalités, qui les confond toutes, jusqu'à ne plus en avoir. Un son sans ton, qui apparaît à l'oreille comme un chuchotement monocorde, ou comme le grésillement de l'appareil de télévi- sion réglé sur un poste mort. Un son qui ne veut plus rien dire.[17] La vidéo est un son, a bien saisi Debray:

Nous étions devant l'image, nous sommes dans le visuel. La forme-flux n'est plus une forme à contempler mais un parasite en fond: le bruit des yeux. Tout le paradoxe de notre troisième âge réside en ceci qu'il donne la suprématie à l'ouïe, et fait du regard une modalité de l'écoute. (Vie et mort de l'image 298)

Et c'est dans cette confusion des sens, dans ce flux continu de messages, que la vie à Blacksmith, USA, se déroule. Du fait de son omniprésence, de son insistance, de la pluralité aussi de ses modes de transmission, télé-radiotabloïds-émissions de toutes sortes, elle devient sa propre réalité. Un événement n'existe plus en soi, du fait de son déroulement, de son actualité, de l'énergie dépensée à sa réalisation, mais du fait de sa diffusion, de sa communication. S'il n'a pas intégré le domaine de la vidéosphère, s'il ne s'est pas transformé en charge électrique et électronique, il n'existe tout simplement pas. C'est l'information qui fait l'événement, sa divulgation et non son apparition. Comme le dit Debray, "Quand la réalité de l'événement a 
pour critère objectif l'avènement de sa trace, l'événement devient la trace elle-même" (Vie et mort 296).

Or, ce rapport au réel nécessairement médiatisé et subordonné à sa reprise électronique est une des régularités du roman. Rien ne se passe qui n'a pas été d'abord annoncé dans les médias. Ni la pluie, qui n'a le droit de tomber que si elle a été dûment annoncée, ni les accidents d'avion qui perdent leur raison d'être en l'absence de reportage. Ainsi, dès le sixième chapitre du livre, un curieux dialogue réunit Gladney et son fils Heinrich. Ils sont en voiture et le père commence par constater qu'il pleut. Des gouttes de pluie tombent tout bonnement sur le pare-brise. Mais cela ne convainc pas Heinrich qui a entendu à la radio qu'il ne devait pleuvoir que plus tard en soirée. S'ensuit un échange sur l'existence même de la pluie, que le fils ne veut pas admettre parce qu'elle n'a pas été annoncée. Il ne faut pas se fier à ses propres sens, dit-il, il n'y a aucune certitude, ajoute-t-il, la vérité est une chose éminemment relative, qui dépend des cadres de référence choisis et dont il faut se méfier, conclut-il. Tout aurait été différent cependant si la pluie avait respecté l'horaire annoncé. Les sens et la radio se seraient complétés pour assurer que c'est bel et bien de la pluie qui tombe. Ainsi, toute la seconde partie du roman est une démonstration de la mainmise des médias dans la vidéosphère, en période de crise comme en temps normal. Un accident grave, un déraillement libère un produit hautement toxique dans l'air, en forme de gros nuage, le Nyodene D. Or, toutes les facettes de ce désastre écologique seront marquées par la médiation nécessaire de sa mise en ondes et en discours. L'événement, initialement, connaîtra diverses désignations selon les bulletins de nouvelles, passant de volutes ("feathery plumes"), et d'épais nuage noir ("black billowing cloud"), à un événement toxique aérien ("airborne toxic event"), syntagme qui sera finalement adopté. L'événement se construit d'une expression à l'autre. Si, au début, il pouvait être écarté, en raison du caractère bénin de l'expression qui le désigne, par la suite il s'impose comme menace, comme danger toxique. Personne ne connaît les conséquences véritables d'une intoxication au Nyodene D, aussi la liste des symptômes déclarés varie-t-elle beaucoup d'un moment à l'autre. Or, les membres de la famille de Gladney présentent ces symptômes dès qu'ils les entendent à la radio, quelle que soit leur validité. Réaction psychosomatique peut-être normale dans les circonstances, mais symptomatique de la médiasphère à l'œuvre.

Finalement, la ville doit être évacuée. Gladney et sa famille doivent se rendre à un camp scout. Là, ils sont pris en charge par une équipe, dont les membres sont en uniforme et portent tous un brassard sur lequel est écrit en majuscule le mot "SIMUVAC". Quand Gladney s'informe de la signification du brassard, il apprend que c'est l'abréviation de "simulated evacuation". L'équipe en place est la même qui commande les exercices d'évacuation en ville et dans les écoles. Gladney est stupéfait. Il explique que l'évacuation n'est pas simulée, qu'elle est vraie. On lui répond qu'on a quand même pensé s'en servir comme d'un modèle. "A form of practice?" dira Gladney. "Are you saying you saw a chance to use the real event in order to rehearse the simulation?" (139). À elle seule, la remarque résume le 
rapport au réel à l'œuvre dans la vidéosphère. L'événement, la véritable évacuation, ne peut être appréhendée que par sa simulation, sa représentation. Et encore, cette représentation est plus importante que le réel, puisqu'elle s'en sert comme d'un moyen pour arriver à ses fins. Le simulacre est l'étalon de l'événement, et il peut seul en prendre la mesure.

De plus, quand les évacués sont finalement transportés à Iron City, où la famille Gladney passera au moins neuf jours, avec 40 autres familles dans un studio de karaté abandonné, la grogne commence à s'installer. Il n'y a presque rien de l'événement toxique à la télévision. C'est comme s'il n'avait jamais existé: pas une image, pas une seule équipe vidéo dépêchée sur les lieux. Les peurs et les sacrifices de ces familles ne veulent rien dire. Leur expérience est complètement dévaluée, neutralisée, disqualifiée par le silence. L'événement ou le réel n'existent, dans ce régime vidéotique, que s'ils sont mis en ondes, qu'en tant qu'ils participent à une transmission. Si un discours ne les a pas saisis, leur valeur est nulle et ils perdent toute existence. C'est la rançon de la vidéosphère.

C'est exactement la même chose qui se passe avec un accident d'avion évité de justesse. Faute de médias, l'expérience perd toute signification. Jack Gladney, qui va chercher sa fille à l'aéroport d'Iron City, assiste à l'arrivée d'un groupe de passagers qui sont dans un état d'excitation avancé. Il semble qu'ils aient tout juste évité l'écrasement. Leur avion avait perdu ses trois moteurs en plein vol, avait commencé à chuter et ce n'est qu'au dernier instant que la situation s'était rétablie. Au début de la chute, ç'avait été la cohue: les passagers et les membres de l'équipage paniquaient, les repas, les accessoires et tous les objets personnels s'étaient retrouvés sens dessus dessous dans les allées. À un moment donné, pourtant, le calme fut rétabli. Des membres de l'équipage décidèrent de faire mine que ce n'était pas un écrasement, "a plane crash", qu'ils allaient subir, mais un atterrissage forcé, "a plane crash landing". L'ajout de ce seul mot fit toute la différence. Les voyageurs se calmèrent et commencèrent à se préparer pour l'atterrissage. Comme si l'addition d'un simple mot avait suffi pour changer la réalité, en modifier le cours. Par la suite, quand la fille de Gladney rejoint enfin son père, elle commente ces événements. Surprise de l'absence de média à l'aéroport, elle apprend qu'il n'y a pas de poste de télévision dans les parages. Elle ne peut alors s'empêcher de conclure, laconiquement, qu'ils ont vécu tout cela pour rien (89-92). Encore une fois, non seulement les mots changent-ils la réalité - -- une leçon que Benjamin Lee Worf avait déjà apprise -- mais les événements eux-mêmes n'ont de valeur, et par suite d'existence, que s'ils sont diffusés.

\section{Cellar door ou lire à travers le bruit}


Si le roman représente la médiatisation sous toutes ses coutures et sutures, s'il l'inscrit comme modalité fondamentale de cet univers, il tente aussi d'en reproduire les effets en initiant une situation de lecture qui se déroulerait sur ce mode un peu particulier configuré comme une relation vidéosphérique. À la lecture aussi, le regard semble être une modalité de l'écoute.

La fragmentation du texte en chapitres courts et sans liens apparents, l'absence d'une intrigue forte, totalisante, remplacée en fait par une logique de l'anecdotique, l'importance des dialogues, souvent sans queue ni tête, d'un quotidien fait de discontinuités, de contradictions et de ratures, l'investissement de lieux publics tels que le centre d'achat, le supermarché, l'aéroport, la voiture, avec leur tonalité et ambiance, tout comme la reprise de clichés publicitaires font en sorte qu'ils reproduisent, à même le roman, la forme et le style des médias. Certains dialogues sont faits, par exemple, d'une incessante dérive des thèmes, d'une succession d'informations discréditées au fur et à mesure qu'elles apparaissent. Une conversation particulièrement tumultueuse survient en auto, lorsque toute la famille est en route vers un des centres d'achat de la région. Le sujet initial est le Dylar et la dérive qui s'ensuit, un modèle du genre:

"What do you know about Dylar?"

"Is that the black girl who's staying with the Stovers?"

"That's Dakar", Steffie said.

"Dakar isn't her name, it's where she's from", Denise said. "It's a country on the ivory coast of Africa".

"The capital is Lagos", Babette said. "I know that because of a surfer movie I saw once where they travel all over the world".

"The Perfect Wave", Heinrich said. "I saw it on TV".

"But what's the girl's name?" Steffie said.

"I don't know", Babette said, "but the movie wasn't called The Perfect Wave. The perfect wave is what they were looking for". 
"If she's an African", Steffie said, "I wonder if she ever rode a camel".

"Try an Audi Turbo".

"Try a Toyota Supra".

"What is it camels store in their humps?" Babette said. "Food or water? I could never get that straight". (80-1)

Et cela continue ainsi pendant un certain temps, la discussion rejoignant le Pérou et les lamas, la population de la Bolivie, etc. À la lecture, l'échange se présente comme un collier de phrases qui se sont agglutinées au hasard des associations, des ressemblances, des kilomètres franchis sur l'autoroute. Il n'y a pas de principe de coopération ni de pertinence qui vaille. C'est le règne de l'anecdotique. Chacun y va de sa suggestion et les cadres de référence ne se recoupent pas. Progresser d'une parole à l'autre, c'est subir une sorte de bruit, une interférence qui camoufle le murmure de la question initiale, jamais répondue. On n'apprend rien sinon, à un second degré, qu'on n'a rien à y apprendre. Il n'y a pas de sens ni de direction: l'autoroute ne mène nulle part, là où se rend justement l'échange. Et la leçon vaut pour tous: "The family is the craddle of the world's misinformation. There must be something in family life that generates factual error. Overcloseness, the noise and heat of being" (81). La famille est source d'entropie, d'une perte d'information; et c'est ce qui nous est transmis, la perte, le glissement, des données dont nous ne pouvons garantir la vérité, mais qui s'imposent du fait qu'elles sont les seules présentes. Une lecture en forme de long dérapage le long de l'autoroute de la désinformation.[18]

De la même façon, les principales sources de savoir du roman sont les émissions de radio, de télévision, les tabloïds, comme le National Inquirer ou Examiner, le Star, le World ou le Globe. La vie après la mort, la présence des extra-terrestres, les complots de toutes sortes, dont l'assassinat d'Elvis, de Marilyn Monroe et de Howard Hughes, tués pour avoir osé convoiter le Saint Suaire de Turin et ses soi-disant pouvoirs régénérateurs; ces fabrications sont accréditées et acceptées au même titre que le reste. Et pour cause: les tabloïds sont la preuve que la vidéosphère est sa propre réalité, qu'elle n'a pas besoin du réel pour diffuser de l'information, pour communiquer. Notre rapport aux études hitlériennes de Gladney est aussi celui qu'un abonné à La sélection du Reader's Digest connaît bien: une relation superficielle, se limitant à l'anecdotique, à ce qui est mondain dans le scientifique. Nous restons à la surface des choses, sachant qui fait quoi, mais sans savoir au juste comment et ce que cela veut dire. Rien n'est justifié, cela est trop lent de toute façon pour les médias; c'est la répétition qui sert de preuve, et la diversité est le gage le plus éloquent de la qualité de l'information. 
White Noise est un roman bruyant et c'est en cela qu'il reproduit efficacement la vidéosphère. Bruyant non seulement à cause des voix des protagonistes, mais de par l'environnement médiatique et les éclats de sa surproduction sonore. Le texte est ponctué de messages divers, de slogans et de publicités, de noms de produits dont l'apparition est impromptue: entre deux paragraphes, au détour d'un dialogue, comme par enchantement. La source n'est presque jamais connue, les mots semblent surgir sans attaches, loin de toute énonciation, comme des fantômes de phrases, hantant le texte de la même façon que les publicités ponctuent nos vies:

The streets were empty. Along Elm all the stores were dark, the two banks were dimly lit, the neon spectacles in the window of the optical shop cast a gimmicky light on the sidewalk.

Dacron, Orlon, Lycra Spandex.

"I know I forget things", she said, "but I didn't know it was so obvious".

"It isn't". (52)

It was here that we met up with the group from the Kung Fu Palace. Tooting horns, waving children. Like wagon trains converging on the Santa Fe Trail. The cloud still hung in the rearview mirror.

Krylon, Rust-Oleum, Red Devil.

We reached Iron City at dawn. There were checkpoints at all the road exists. (159)

Dans les deux cas, l'apparition de ces noms de produits, dans des phrases sans verbe, sans déictique, n'est nullement motivée. Elle est pure irruption, comme si le texte avait happé, par mégarde, des sons présents dans l'environnement, ces images acoustiques étant le signe d'une prise directe de la narration sur ce monde. Ces phrases, pourtant, ne perturbent pas le récit, les personnages ne semblent même pas s'en rendre compte. Elles apparaissent et disparaissent sans que personne ne bronche, sauf le lecteur qui doit les assimiler ou, à défaut, s'en accommoder. Il devra répéter l'exercice assez souvent, le texte résonnant régulièrement de ces énumérations de produits qui se suivent comme des formules sacrées: "Kleenex Softique. Kleenex Softique" (39), "Mastercard, Visa, American Express" (100), "Toyota Celica" (155), "Dristan Ultra, Dristan Ultra" (167), 
"Clorets, Velamints, Freedent" (229), "Panasonic" (241), "Tegrin, Denorex, Selsun Blue" (289), "Random Access Memory, Acquired Immune Deficiency Syndrome, Mutual Assured Destruction" (303), etc.. Comme l'explique Murray, il y a dans ces mots, ces slogans, ces publicités, une masse inouie d'informations. Mais, il faut regarder au-delà des messages grossièrement codés et des répétitions enfantines, au-delà de notre irritation, lassitude ou dégoût et prendre ces lexies comme des mantra: "Coke is it. Coke is it" (51). Ces mots qui flottent dans le texte forment un chant, destiné à nous faire passer à un autre niveau de conscience, une sorte de mysticisme informatique et vidéosphérique.[19] Le choix d'une génération.

La plupart du temps, les énoncés apparaissent comme de pures émanations de l'environnement. Ils sont là, comme les murmures de la machine, ces bruits qui nous accompagnent et dont nous apprenons vite à ne plus nous soucier. Quelquefois cependant, la source de ce chant est connue: ici la télévision, la radio, les haut-parleurs d'un centre d'achat, là une femme qui marche dans la rue et qui énumère: "A decongestant, an antihistamine, a cough /pp. 24-25/ suppressant, a pain reliever" (262). Il y a constamment un poste de télé ou de radio qui clame et qui dit, qui transmet son savoir incontournable, comme dans une toile hyper-réaliste:

The TV said: "And other trends that could dramatically impact your portfolio". (61)

The radio said: "It's the rainbow hologram that gives this credit card a marketing intrigue". (122)

The voice at the end of the bed said: "Meanwhile here is a quick and attractive lemon garnish suitable for any seafood". (178)

Le texte semble n'avoir capté et gardé que des fragments de messages, comme s'il s'agissait là du résultat d'une écoute distraite qui, attirée par une sonorité particulière, aurait saisi quelques mots, une phrase, une parcelle de savoir. L'information est tout aussi inutile qu'inutilisable, pourtant on en reconnaît vite la provenance, l'attache, le produit: publicité pour des fonds mutuels ou pour une compagnie de cartes de crédit, segment d'une émission d'informations ménagères. Ces messages tronqués ne veulent rien dire (de précis), mais leur présence parle et elle nous rappelle où nous sommes. Le roman nous force à écouter ces slogans, à les reconnaître, à les retrouver là où on ne les attendait plus, là où on pouvait même s'en croire à l'abri. Il nous renvoie à nous-même. Il ne se lit pas, il s'inhale, comme l'air ambiant. [20] 
À l'instar des productions américaines récentes de littérature populaire où de la publicité est insérée au milieu du texte, mais en poussant la logique encore plus loin car plus rien ne sépare le texte du roman de celui de la publicité, White Noise nous amène à moduler notre lecture comme si elle n'était rien d'autre qu'une forme dérivée de la vidéosphère, participant du même régime médiatique. Sa lecture n'oppose plus un mode de transmission à un autre, une médiasphère à une autre; mais, par le jeu des vases communiquants, elle réussit à établir un pont entre les deux en reproduisant les manières, le style, l'expérience de l'une dans l'autre.

Lire White Noise, c'est se retrouver comme dans le monde, au lieu de s'en isoler, $c^{\prime}$ est retrouver le même, à son tour reproduit: les mêmes tensions, les mêmes insécurités. Pas étonnant que la critique postmoderne, qui s'est emparée du livre, y ait trouvé écho à ses principales préoccupations. Il y a entre le livre et la vie une correspondance qui favorise l'amalgame des deux dans une sphère où les procédés et les régularités autorisent les lecteurs avertis à y ressentir les effets escomptés. La convergence interprétative notée repose sur un consensus, le partage d'un savoir, dont les diverses traductions -- narratives, critiques, théoriques -- assurent la diffusion et l'implantation. On s'entend donc sur le fait qu'il n'y a plus rien à entendre, que le bruit s'est répandu partout, nous empêchant de comprendre ce qui pourrait être dit, ce qui subsistera peut-être quand l'Histoire reprendra son cours en s'engageant dans une nouvelle période, une autre conception du monde et de ses rapports sociaux, économiques et politiques. On s'entend sur la façon de représenter nos vies, de les mettre en récit et d'en faire des intrigues, de les rendre vraisemblables, malgré leurs nombreuses incohérences, et de les interpréter. On s'entend aussi sur la façon dont les médias ont réussi à s'immiscer dans nos vies, dans nos activités les plus intimes, telle la lecture.

White Noise dit: "Soyez à l'écoute du Dylar"; et c'est bien la possibilité d'en reconnaître la mélodie qui nous effraie le plus.

\section{Bertrand Gervais \\ Département d'Études littéraires Université du Québec à Montréal}

Surface Page d'Acceuil/Home Page 
[1]Don DeLillo, White Noise (New York: Penguin Books, 1984). Les prochaines références à ce roman seront faites, entre parenthèses, dans le corps du texte.

[2]John G. Cawelti, Adventure, Mystery, and Romance (Chicago: The University of Chicago Press, 1976).

[3]Franck Lentricchia, éd., New Essays on White Noise (New York:

Cambridge University Press, 1991).

[4]Paula Bryant, "Extending the Fabulative Continuum: DeLillo, Mooney, and Federman," Extrapolation 30:2 (1989): 156-165.

[5]Michael Messmer, "Thinking it Through Completely: the Interpretation of Nucleart Culture," The Centennial Review 32;4 (1988): 397-413.

[6]Noel King, "Reading White Noise: Floating Remarks," Critical Quaterly 33;3 (automne 1991): 69.

[7]Tom LeClair, In the Loop (Chicago et Urbana: University of Illinois Press, 1987).

[8]Robert Dion, "Une critique du postmoderne," Tangence 39:88.

[9]Stanley Fish, Is There a Text in This Class? (Cambridge et Londres: Harvard University Press, 1982): 171.

[10]Pour Paul A. Cantor, dont je reprends ici les propos, l'histoire apparaît dans le texte comme une sorte de musée ou, mieux encore, comme un centre d'achat des possibilités humaines. ("Adolf, We Hardly Knew You," New Essays on White Noise, p. 41). Il dit, de plus, que si le modernisme se percevait comme la fin de l'histoire puisqu'il s'en croyait l'apogée, le postmodernisme serait un moment posthistorique, qui intègre l'histoire pour mieux la désarmer, la rendre inoffensive, inopérante.

[11]Malgré son propre éclectisme, il s'étonnera, en début de roman, des études menées dans le département d'"american environments": "I understand the music. I understand the movies, I even see how comic books can tell us things. But there are full professors in this place who read nothing but cereal boxes"(10).

[12]Cantor dira ainsi: "we ought to recognize that White Noise is not itself an example of Hitler studies, but rather a novel which portrays a professor 
involved in Hitler studies. DeLillo may be trying to characterize the contemporary world by showing that such a phenomenon as Hitler studies has become possible in it. In fact, DeLillo could find no better example of the flattening-out of contemporary existence than the routinization of Hitler's charisma at the College-on-the-Hill. Like all of DeLillo's work, White Noise portrays postmodern America. " ("Adolf, We Hardly Knew You," loc. cit., p. 41)

[13]Dans cette logique, Gladney baptisera son fils Heinrich, parce qu'un nom allemand est un signe d'autorité et de force. Un nom allemand impressionne: "There's something about German names, the German language, German things. I don't know what exactly. It's just there. In the middle of it all is Hitler, of course"(63).

[14]Régis Debray, Cours de médiologie générale (Paris: Gallimard, 1991); Vie et mort de l'image. Une histoire du regard en occident (Paris: Gallimard, 1992; L'état séducteur. Les révolutions médiologiques du pouvoir (Paris: Gallimard, 1993).

[15]Les liens entre vidéosphère, guerre froide et aliénation, qui sont inscrits en toutes lettres dansWhite Noise et qui donnent au roman sa force, avaient déjà connus une première formulation dans un roman d'anticipation de Ray Bradbury, publié en 1953, Farhenheit 451. Des télévisions à écrans multiples, qui simulent et stimulent l'interaction, sans aucun effort de la part du spectateur, ont envahi les salons. Le spectateur est appelé à participer à l'histoire, à donner la réplique aux personnages qui lui adressent la parole et qui semblent le regarder. Cela le tient occupé. Pour le tenir tranquille, pour l'empêcher de se faire du mauvais sang, et pour le retenir sagement à la maison, ignorant des machinations de l'Etat, de nombreuses drogues lui sont de plus accessibles. Quelques trente ans plus tard, les principaux traits de l'anticipation de Bradbury s'imposent, mais cette fois-ci comme univers fictionnel vraisemblable, crédible.

[16]Debray,Vie et mort de l'image 297 et passim.

[17]Leonard Wilcox dit à cet effet, "The very notion of "white noise" that is so central to the novel implies a neutral and reified mediaspeech, but also a surplus of data and an entropic blanket of information glut which flows from a media-saturated society." ("Baudrillard, DeLillo's White Noise, and the End of Heroic Narrative," Contemporary Literature 32;3 (automne 1991): 347.

[18]Noel King, qui analyse le même passage, décrit bien cette systématique de l'erreur: "The comic device consists in producing a series of statements which are "correct" to the extent that they identify "error" in a previous statement or assertion but which at the same time, in that very act of correction, introduce a further factor of error, presenting different kinds of 
error, thereby effecting an exponential multiplication of misinformation." ("Reading White Noise" 73)

[19]DeLillo, dans une entrevue plus récente s'expliquait sur son utilisation des noms de produits: "There's something nearly mystical about certain words and phrases that float through our lives. It's computer mysticism. Words that are computer generated to be used on products that might be sold anywhere from Japan to Denmark--words devised to be pronounceable in a hundred languages. And when you detach one of these words from the product it was designed to serve, the word acquires a chantlike quality. Years ago somebody decided--I don't know how this conclusion was reached--that the most beautiful phrase in the English language was cellar door. If you concentrate on the sound, if you disassociate the words from the object they denote, and if you say the words over and over, they become a sort of higher Esperanto. This is how Toyota Celica began its life. It was pure chant at the beginning. Then they had to find an object to accommodate the words." ("Interview. The Art of Fiction CXXXV," The Paris Review 128 (automne 1993): 291.

[20]A ce sujet, justement, Franck Lentricchia signalait, dans son introduction aux New Essays on White Noise, qu'un de ses étudiants lui a dit qu'il n'avait pas lu le texte, ce serait trop simple, qu'il l'avait plutôt inhalé (p. 7). 